\title{
WATER CONTENT OF STRYCHNOS GERRARDII DETERMINES VITRIFICATION IN CRYOPRESERVATION: BIOPHYSICAL APPROACHES
}

\author{
Aline Schneider Teixeira ${ }^{1,2^{*}}$, Boby Varghese ${ }^{1}$, Antonio Diego Molina-Garcia ${ }^{3}$, Norman W. \\ Pammenter ${ }^{1}$, Patricia Berjak (Deceased) ${ }^{1}$ \\ ${ }^{1}$ Plant Germplasm Conservation Research, School of Life Sciences, University of KwaZulu-Natal, \\ Durban, South Africa; ${ }^{2}$ Current address: Centro de Investigación y Desarrollo en Criotecnología \\ de los Alimentos (CIDCA-CONICET), La Plata, Argentina; ${ }^{3}$ Instituto Ciencia y Tecnología de \\ Alimentos y Nutrición (ICTAN-CSIC), Madrid, Spain.
}

Corresponding author: * teixeiraline@hotmail.com

Vitrification is an effective freeze-avoidance mechanism and living tissue cryopreservation, in most cases, relies on it. As a glass is exceedingly viscous and stops all chemical reactions that require molecular diffusion, its formation leads to metabolic inactivity and stability over time. However, the various procedures (prior to, and immediately after) ultra-low temperature storage generate reactive oxygen species (ROS) which often preclude success, in most cases associated with lethal effects on the shoot apical meristem.

Testing the success of new cryopreservation protocols, or their adaptation to different species, varieties or tissues, by checking the actual recovery after cryostorage, is long, costly and tedious. Not only is a considerable time involved, but for obtaining statistically significant data, a large number of specimens must be employed. To check for successful vitrification, simplified biophysical approaches can reduce length and cost of the study.

In order to check the effect of cathodic water (a treatment proposed to reduce oxidative damages) on the correct vitrification of Strychnos gerrardii, zygotic axes submitted to a frequently employed cryopreservation protocol were monitored through its different stages by low-temperature scanning electron microscopy (cryo-SEM) and differential scanning calorimetry (DSC). Axes were immersed for $30 \mathrm{~min}$ in cathodic water following excision and after the dehydration stage in the protocol. DSC permitted the quantification of the frozen water content reduction and the detection of the eventual glass transition. In parallel, the vitrification of the cellular content was ascertained by cryo-SEM.

Cathodic water treatments were shown not to affect negatively the Strychnos gerrardii zygotic axes vitrification. Results show how tissues at intermediate treatment steps (with decreasing water content) develop ice crystals during liquid nitrogen cooling, while tissue vitrification is successfully achieved after the final protocol stage.

Source of Funding: This work has been carried out thanks in a project "Elucidating the mode of action of cathodic water in ameliorating oxidative damage associated with explant processing for cryopreservation". A. Schneider Teixeira was supported by a University of Kwazulu-Natal, postdoctoral scholarship.

Conflict of Interest: None to disclose 